\title{
Low-fluoroscopy atrial fibrillation ablation with contact force and ultrasound technologies: a learning curve
}

This article was published in the following Dove Medical Press journal: Pragmatic and Observational Research

\author{
Paul C Zei' \\ Tina D Hunter ${ }^{2}$ \\ Larry M Gache ${ }^{2}$ \\ Gerri O'Riordan ${ }^{3}$ \\ Tina Baykaner ${ }^{3}$ \\ Chad R Brodt ${ }^{3}$ \\ 'Cardiac Electrophysiology, Brigham \\ and Women's Hospital, Boston, MA, \\ USA; ${ }^{2}$ Real World Evidence, CTI \\ Clinical Trial and Consulting Services, \\ Covington, KY, USA; ${ }^{3}$ Cardiovascular \\ Medicine, Stanford University, \\ Stanford, CA, USA
}

Correspondence: Tina D Hunter Real World Evidence, CTI Clinical Trial and Consulting Services, 100 East River Center Boulevard, Covington, KY $4 \mathrm{I} 0 \mathrm{II}$, USA

Tel +I 5135989290

Email thunter@ctifacts.com
Background: Fluoroscopy exposure during catheter ablation is a health hazard to patients and operators. This study presents the results of implementing a low-fluoroscopy workflow using modern contact force (CF) technologies in paroxysmal atrial fibrillation (PAF) ablation.

Methods: A fluoroscopy reduction workflow was implemented and subsequent catheter ablations for PAF were evaluated. After vascular access with ultrasound guidance, a THERMOCOOL SMARTTOUCH ${ }^{\circledR}$ Catheter (ST) was advanced into the right atrium. The decapolar catheter was placed without fluoroscopy. A double-transseptal puncture was performed under intracardiac echocardiography guidance. ST and mapping catheters were advanced into the left atrium. A left atrial map was created, and pulmonary vein (PV) isolation was confirmed via entrance and exit block before and after the administration of isoproterenol or adenosine.

Results: Forty-three patients underwent PAF ablation with fluoroscopy reduction workflow (mean age: $66 \pm 9$ years; $70 \%$ male), performed by five operators. Acute success rate (PV isolation) was $96.5 \%$ of PVs. One case of pericardial effusion, not requiring intervention, was the only acute complication. Mean procedure time was $217 \pm 42$ minutes. Mean fluoroscopy time was $2.3 \pm 3.0$ minutes, with $97.7 \%$ of patients having $<10$ minutes and $86.0 \%$ having $<5$ minutes. A significant downward trend over time was observed, suggesting a rapid learning curve for fluoroscopy reduction. Freedom from any atrial arrhythmias without reablation was $80.0 \%$ after a mean follow-up of $12 \pm 3$ months.

Conclusion: Low fluoroscopy time is achievable with CF technologies after a short learning curve, without compromising patient safety or effectiveness.

Keywords: atrial fibrillation, catheter ablation, workflow, contact force, low fluoroscopy

\section{Introduction}

Contact force (CF)-sensing catheters have been shown to improve procedural efficiency and effectiveness during ablation for paroxysmal atrial fibrillation (PAF). ${ }^{1} \mathrm{CF}$ catheters have a force-sensing mechanism incorporated into the distal tip of the catheter, allowing real-time assessment of catheter-tissue contact. A recent meta-analysis suggests that the use of CF-sensing technology reduced risk of atrial fibrillation (AF) recurrence. ${ }^{2}$ One important procedural benefit of CF-sensing catheters is reduced fluoroscopy times and radiation doses, ${ }^{3-12}$ a concern for both patients and health care professionals alike. Cumulative radiation exposure among operators is a long-term health hazard, ${ }^{13,14}$ while high-fluoroscopy exposure among patients likely confers an increased risk of radiationinduced complications. ${ }^{15,16}$ Several recent studies have reported reductions in fluoroscopy with $\mathrm{CF}$-sensing catheters compared to pre-CF radiofrequency (RF) catheters, with 
mean fluoroscopy times of 9.5-19.4 minutes reported in the CF cohorts vs 40.7-41 minutes in the non-CF cohorts. ${ }^{4,12}$ Researchers have recognized a learning curve, whereby introducing CF-sensing catheters reduces median fluoroscopy times progressively over time. ${ }^{4,17}$ These reductions in fluoroscopy time appear to be independent of procedure complexity (PAF vs persistent AF) and approach (de novo vs reablation).

Despite the evidence demonstrating lower fluoroscopy times associated with CF-sensing catheters, the learning curve pertaining to the low-fluoroscopy workflow has not been described extensively, particularly in the initial procedures following introduction of the technology. Thus, the primary aim of this study was to confirm and characterize the learning curve for a low-fluoroscopy workflow using a CF catheter and stability technologies in PAF ablation. Consistent with previous studies, we hypothesized that implementation of the new workflow would result in a learning curve of progressively reduced fluoroscopy times. In addition, we sought to determine whether the new low-fluoroscopy workflow could be achieved without compromising safety or effectiveness. Therefore, we conducted exploratory analyses of effectiveness endpoints, notably, freedom from recurrence of atrial arrhythmias within the 12 months of study follow-up.

\section{Methods}

This study analyzed real-world data collected at a single high-volume academic electrophysiology (EP) practice with fellows actively participating in the procedures between July 2014 and December 2015. Ablations were performed by five operators. Typically, the primary operator was the attending physician, with fellows providing limited assistance. The study population consisted of consecutive symptomatic, drug-refractory PAF patients presenting for catheter ablation. All patients met accepted guideline indications for catheter ablation. ${ }^{18}$ Ablations were performed with the THERMOCOOL SMARTTOUCH ${ }^{\circledR}$ Catheter (ST) (Biosense Webster, Inc., Irvine, CA, USA) and CARTO VISITAG ${ }^{\mathrm{TM}}$ Module (Visitag) (Biosense Webster, Inc.). The details of the standardized workflow utilizing this CF-sensing technology are presented below. Study data consist of baseline patient characteristics, comorbidities, procedural details, complications, and effectiveness outcomes.

The Stanford University Research Compliance Office approved this study and granted a waiver of informed consent for analysis and publication of de-identified records on November 1, 2016. All data used to perform the statistical analysis were de-identified and accessed in compliance with the Health Insurance Portability and Accountability Act.
This research was conducted in conformity with the ethical standards of the Declaration of Helsinki.

\section{Workflow for ST ablations}

After informed consent and induction of general anesthesia, a multi-electrode temperature probe (CIRCA S-CATH ${ }^{\mathrm{TM}}$, CIRCA Scientific, LLC, Englewood, CO, USA) was advanced into the esophagus. Vascular access was obtained under ultrasound guidance. An intracardiac echocardiography (ICE) catheter (ACUNAVTM Catheter or SOUNDSTAR ${ }^{\circledR}$ Catheter, Biosense Webster, Inc.) was advanced using progressively reduced fluoroscopic guidance over the evaluation period. An ST ablation catheter was inserted into the right atrium (RA), also with a continuing decrease in fluoroscopic guidance as the study period progressed. An electroanatomic (EA) map and shell of the RA were created using the ablation catheter, and a decapolar catheter was advanced using EA map guidance into the coronary sinus. Double-transseptal puncture was performed using ICE guidance and also required progressively less fluoroscopy over time.

A multi-electrode mapping catheter $\left(\mathrm{LASSO}^{\circledR}\right.$ Catheter or PENTARAYTM Catheter, Biosense Webster, Inc.) and the ablation catheter were advanced into the left atrium (LA). An EA map and shell of the LA was then created using a combination of these catheters. The phrenic nerve and esophagus course, as delineated by the esophageal temperature probe, were marked on the EA map. Wide-area circumferential pulmonary vein (PV) antral ablation was then used to create electrical isolation of all PVs. RF delivery was continuous if possible, and the ablation catheter was moved in a contiguous fashion once ablation parameters that resulted in automated tagging of points using the Visitag Module were achieved. Typically, parameters for Visitag were set at a minimum $\mathrm{CF}$ of $5 \mathrm{~g}$ for a minimum of $45 \%$ of the time with a maximum location stability of 2-mm and minimum time of 3 seconds.

Electrical isolation of each PV was confirmed with both entrance and exit block in sinus rhythm, after a minimum 20-minute waiting period post-ablation. Isolation was confirmed with and without either isoproterenol infusion of up to $20 \mu \mathrm{g} / \mathrm{min}$ or adenosine via central venous access. Any evidence of electrical reconnection was treated with additional ablation until electrical isolation was achieved. If excessively rapid increases or high elevation of temperatures were measured with the esophageal temperature probe, additional ablation along the corresponding portion of the posterior wall was aborted. Both the ablation and PENTARAYTM Catheters were manipulated during mapping and ablation with minimal to no fluoroscopy, relying on the mapping system and ICE 
for guidance of catheter movement. All procedures were performed with a combination of the attending physician and EP fellow manipulating the catheters.

\section{Data collection and follow-up}

Baseline patient characteristics, comorbid conditions, and AF history were recorded prior to ablation. Procedural details, including the ablations performed, total procedure time, total RF time, and fluoroscopy time, were also captured. Procedure time was defined as the period from sheath access through removal of all intracardiac catheters. All patients were monitored on telemetry in a recovery unit overnight.

Patients were followed for safety during the procedure and prior to hospital discharge, and serious procedure-related complications were recorded. These events were defined as outcomes attributable to the procedure that were life-threatening, required further intervention, or delayed discharge due to safety concerns.

Typical follow-up was at 1, 3, 6, and 12 months, then annually thereafter as desired by the patient. Cardiac rhythm, medication usage, and effectiveness measures were collected at a 12-month office visit, ideally conducted within a target period of 12 months \pm 30 days following the procedure. An electrocardiogram was performed at each visit, and a Holter monitor was worn for up to 2 weeks if possible at the 3-, 6-, and 12-month follow-up intervals. For this analysis, we evaluated rhythm status at the 12 -month visit if available, or at the follow-up visit closest to the 12-month target window. Patients were typically maintained on the same antiarrhythmic drugs (AADs), if any, taken prior to the initial ablation procedure, and AAD use was continued for $2-3$ months postablation. After 3 months, patients were advised to discontinue AADs if no evidence of recurrent $\mathrm{AF}$ or other sustained atrial tachyarrhythmia was seen. However, patients who wished to continue using AADs could do so. The primary effectiveness endpoint was freedom from recurrence of atrial arrhythmias at the 12-month follow-up visit. Freedom from recurrence was defined as being in sinus rhythm at the 12-month followup visit without an intervening ablation.

\section{Statistical analysis}

All available patient characteristics, procedural details, complications, and effectiveness measures were summarized using descriptive statistics. Procedure, RF, and fluoroscopy times were modeled using linear regression, and the recurrence endpoint was modeled with logistic regression. Patient characteristics, procedure chronology, procedural details, and follow-up time were tested as explanatory variables in all models. Procedure chronology, type of ablation (de novo vs reablation), additional AF ablation targets (yes/ no), and ablation for additional arrhythmias (yes/no) were of primary interest.

All statistical analyses in this study were performed using SAS software, Version 9.2 or higher (SAS Institute Inc., Cary, NC, USA).

\section{Results}

Forty-three patients met the inclusion criterion of undergoing ablation for PAF during the study period, with 35 patients having follow-up information. The average time to follow-up for these patients was $12 \pm 3$ months.

\section{Patient characteristics}

Baseline patient characteristics are shown in Table 1. The majority of the study population was male $(69.8 \%)$ and the average age was $66 \pm 8.6$ years. The median time since $\mathrm{AF}$ diagnosis was 60 months (IQR: $24-96$ months) and 58.1\% of the patients had prior ablations. Hypertension was the most

Table I Baseline patient characteristics

\begin{tabular}{|l|l|l|}
\hline & N & $\%$ \\
\hline Total patients & 43 & 100 \\
\hline Gender, male & 30 & 69.8 \\
\hline Had prior AF ablation(s) & 25 & 58.1 \\
\hline Had implantable cardiac device & 4 & 9.3 \\
\hline Comorbid conditions & & \\
\hline Congestive heart failure & 7 & 16.3 \\
\hline Coronary artery disease & 5 & 11.6 \\
\hline Diabetes mellitus & 5 & 11.6 \\
\hline Hypertension & 22 & 51.2 \\
\hline Peripheral vascular disease & 1 & 2.3 \\
\hline Sleep apnea & 11 & 25.6 \\
\hline Stroke & 5 & 11.6 \\
\hline Valvular disease & 3 & 7.0 \\
\hline CHA DS - VASc score & & \\
\hline 0 & 2 & 4.7 \\
\hline I & 12 & 27.9 \\
\hline 2 & 13 & 30.2 \\
\hline 3 & 8 & 18.6 \\
\hline 4 & 6 & 14.0 \\
\hline 5 & $\mathrm{I}$ & 2.3 \\
\hline 6 & 0 & 0.0 \\
\hline 7 & $\mathrm{I}$ & 2.3 \\
\hline 8 & 0 & 0.0 \\
\hline & Mean & $\mathbf{S D}$ \\
\hline Age at ablation & 66.3 & 8.6 \\
\hline Months with AF & 77.0 & 89.8 \\
\hline Median, IQR & 60.0 & $24-96$ \\
\hline Ejection fraction (\%) & 53.7 & 3.5 \\
\hline Months to follow-up visit & 11.9 & 3.1 \\
\hline
\end{tabular}

Abbreviation: $\mathrm{AF}$, atrial fibrillation. 
prevalent individual comorbidity, followed by sleep apnea (51.2\% and $25.6 \%$, respectively, Table 1$)$. Most patients had low to moderate risk of stroke, as evidenced by almost two-thirds having $\mathrm{CHA}_{2} \mathrm{DS}_{2}$-VASc scores of $\leq 2$ (Table 1).

\section{Procedural efficiency}

The mean procedure time was $217 \pm 42$ minutes, with a mean RF time of $65 \pm 19$ minutes (Table 2). The mean fluoroscopy time was $2.3 \pm 3.0$ minutes, with $97.7 \%$ of patients having $<10$ minutes and $86.0 \%$ having $<5$ minutes of exposure (Table 2). A clear downward trend over time was observed, suggesting a rapid learning curve for reducing fluoroscopy usage (Figure 1). Median fluoroscopy time per procedure

Table 2 Procedural detail

\begin{tabular}{|l|l|l|}
\hline Efficiency & Mean & SD \\
\hline Total procedure time (minutes) & 217 & 41.9 \\
\hline Total radiofrequency time (minutes) & 65 & 19.4 \\
\hline Total fluoroscopy time (minutes) & 2.3 & 3.0 \\
\hline Low fluoroscopy targets & $\mathbf{n} / \mathbf{N}$ & $\%$ \\
\hline Fluoroscopy time <10 minutes & $42 / 43$ & 97.7 \\
\hline Fluoroscopy time <5 minutes & $37 / 43$ & 86.0 \\
\hline Acute procedural success & $\mathbf{n} / \mathbf{N}$ & $\%$ \\
\hline Pulmonary veins isolated & $166 / 172$ & 96.5 \\
\hline
\end{tabular}

during the second half of the evaluation period was 9 seconds $(n=22)$, reduced from 3.8 minutes during the first half $(n=21)$. After adjusting for statistically significant procedural differences, increasing procedure chronology was shown to be significantly associated with a reduction in fluoroscopy time $(P \leq 0.0001)$, but not with procedure or RF times.

\section{Effectiveness and safety}

Effectiveness measures at the 12-month visit are summarized in Table 3. Freedom from recurrence of any atrial arrhythmia was met in $80.0 \%$ of the population. Thirteen of the 35 patients with follow-up remained on AADs either by choice or to maintain sinus rhythm. At each visit, patients were advised to discontinue AADs if no AF was observed during their evaluation, but many patients elected to continue them. In single-predictor logistic regression models, only the chronology of the ablation (categorized as first $1 / 3$ vs last $2 / 3$ of procedures) was significant in explaining freedom from recurrence at the 12-month follow-up visit $(P=0.03)$. Moreover, the magnitude of the difference in the rates for the two groups was clinically significant, with the last $2 / 3$ of procedures resulting in a higher rate of freedom from recurrence than the first $1 / 3$ procedures $(91.3 \%, \mathrm{n}=23$ vs $58.3 \%, \mathrm{n}=12)$.

SmartTouch PAF cohort: fluoroscopy time vs procedure date (procedures identified by index ablation)

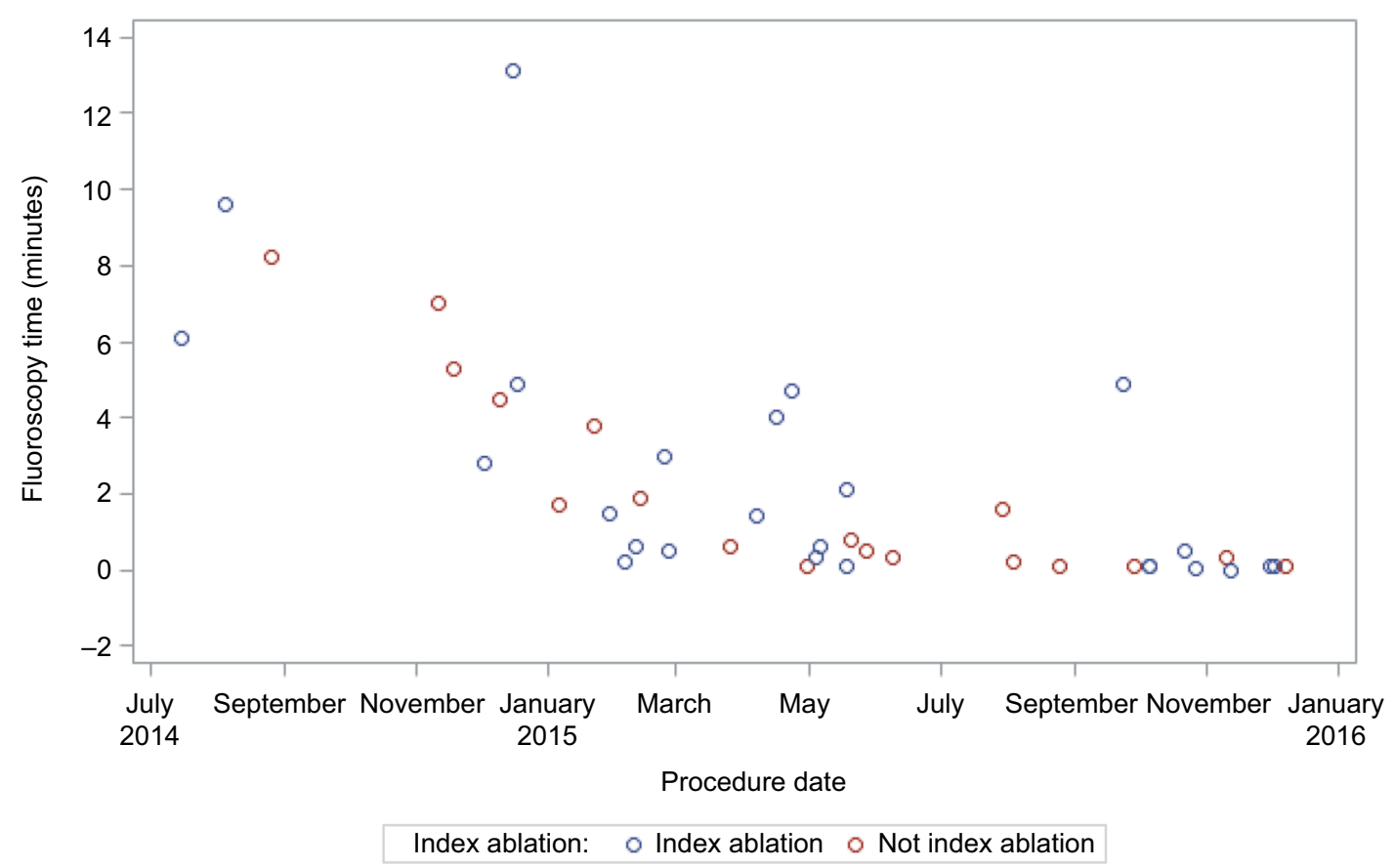

Figure I Reduction of fluoroscopy usage learning curve.

Notes: Median fluoroscopy times: all 43 procedures, 0.6 minutes; first 21 procedures, 3.8 minutes; last 22 procedures, 9 seconds. Abbreviation: PAF, paroxysmal atrial fibrillation. 
Table 3 Twelve-month effectiveness

\begin{tabular}{|l|l|l|}
\hline \multicolumn{1}{|l|}{} & N & $\%$ \\
\hline Total patients & $\mathbf{4 3}$ & $\mathbf{1 0 0}$ \\
\hline Cardiac rhythm at I2-month visit & 32 & 74.4 \\
\hline Sinus rhythm & $\mathrm{I}$ & 2.3 \\
\hline AF - paroxysmal & 2 & 4.7 \\
\hline AF - persistent & 8 & 18.6 \\
\hline No follow-up visit & 22 \\
\hline Antiarrhythmic medication at I2-month visit \\
\hline No & 13 & 51.2 \\
\hline Yes & 8 & 30.2 \\
\hline No follow-up visit & 8 & 18.6 \\
\hline Reablation procedure prior to I2-month visit \\
\hline No & 3 I & 72.1 \\
\hline Yes & 4 \\
\hline No follow-up visit & 8 & 9.3 \\
\hline I2-month primary effectiveness (N=35 patients with follow-up) \\
\hline Freedom from recurrence & $28 / 35$ & 80.0 \\
\hline
\end{tabular}

Abbreviation: $\mathrm{AF}$, atrial fibrillation.

The only serious procedure-related complication in our study population was a case of pericardial effusion that did not require intervention and had no long-term sequelae.

\section{Discussion}

In this academic teaching center, a concerted effort to reduce fluoroscopy utilization during catheter ablation for AF proved to be safe, effective, and achievable, with total fluoroscopy time decreasing dramatically over the study period. During the 18 months immediately following adoption of procedural changes to reduce fluoroscopy, median fluoroscopy time was reduced from $\sim 4$ minutes in the first 21 study procedures to just 9 seconds in the last 22 procedures. This reduction was achieved via a workflow that relied on ultrasound and EA mapping to overcome procedural difficulties that historically relied on fluoroscopy. Specifically, ultrasound was used to facilitate vascular access, EA mapping systems were used to enable diagnostic catheter placement without fluoroscopy, transseptal puncture was accomplished via ICE visualization, and LA mapping and ablation were facilitated with the EA mapping system.

The single complication was well within reported rates for AF ablation, ${ }^{7}$ and overall freedom from $\mathrm{AF}$ at 1 year after ablation was also similar to published rates. ${ }^{1,7}$ In addition to the expected reductions in fluoroscopy over the implementation period for this new low-fluoroscopy workflow, the large increase in effectiveness from the first $1 / 3$ procedures to the later $2 / 3$ procedures was a striking unforeseen benefit.

Technologies that allow for improved confidence in positional relationships between catheters and cardiac structures, including ICE, advanced mapping systems, and CF-sensing catheters may contribute to the ability to safely and effectively achieve a minimal fluoroscopic ablation technique and workflow. In this study, CARTO ${ }^{\mathrm{TM}}$ EA mapping, $\mathrm{CF}$ ablation, and ICE technologies were utilized. Though the adaptability of this workflow to alternative technologies is unclear, other studies of minimal or zero fluoroscopy $\mathrm{AF}$ ablation have also demonstrated safety and acute procedural efficacy, as measured primarily by PV isolation and acute complication rates. ${ }^{19-22}$ To our knowledge, only one other study has reported outcomes during an active learning period for fluoroscopy reduction. ${ }^{4}$ That research found significantly lower fluoroscopy times $(-77 \%)$, radiation doses $(-71 \%)$, and procedural times $(-19 \%)$ with routine use of CF technology. Our study underscores the existence of this learning period, but illustrates that learning can occur soon after the adoption of a low-fluoroscopy workflow, and particularly among just 43 ablation procedures. This learning period occurred in the setting of an academic EP practice, where trainees actively participate in procedures, learning catheter manipulation, mapping, and ablation. Despite the relative inexperience of operators in this setting, safe and effective fluoroscopic reduction was achieved. It will be important to evaluate the feasibility, safety, and efficacy of these fluoroscopy reduction techniques more broadly among the wider EP community.

We surmise that each component of the overall workflow described in our study contributes importantly to the reduction of fluoroscopy utilization while maintaining safety and effectiveness. Groin access via ultrasound visualization is a well-described technique that was utilized in all procedures in our evaluation. ${ }^{23}$ The ability to visualize catheters using a combination of ICE and EA mapping likely provides improved real-time imaging of catheter position in relationship to cardiac structures that is more detailed and accurate than that provided by traditional fluoroscopy. CF-sensing technology may provide additional benefit by ensuring adequate catheter-tissue contact during RF ablation and during catheter manipulation when monitoring for excessive $\mathrm{CF},{ }^{1,24,25}$ which may contribute to a safe and effective reduction in fluoroscopy utilization. Further study will be critical to better understanding the full impacts and potential benefits of these technologies.

\section{Limitations}

The primary limitation of this study is the non-randomized retrospective design. As such, the results lack generalizability beyond the site at which the procedures occurred. Although it is probable that our findings are widely applicable, sitespecific contextual factors could lead to different results across sites or operators. Typical fluoroscopy time at the onset of the study period was already reduced compared to fluoroscopy times reported in the literature, suggesting 
operators had already adopted some techniques to reduce fluoroscopy. Therefore, applicability of these results to centers using significantly higher fluoroscopy per case is unclear. Other limitations include the potential for confounding by unmeasured variables and the possibility of insufficient power to detect significant differences due to low sample size.

\section{Conclusion}

With concerted effort and with the use of a combination of ICE, EA mapping, and CF technologies, effective and significant reduction of fluoroscopic utilization during PAF ablation procedures is achievable while simultaneously maintaining safety and effectiveness.

\section{Acknowledgments}

The authors appreciate the input of Megan Estes, Lee Ming Boo, Michael Fisher, and Amanda Coleman for their assistance in the study and manuscript development process. Analysis and report writing were funded by Biosense Webster, Inc. Ablation procedures and data collection were performed independently by the investigators.

\section{Disclosure}

PC Zei and CR Brodt are recipients of research grants from Biosense Webster, Inc. TD Hunter and LM Gache are employees of CTI Clinical Trial and Consulting Services, which is a consultant to Biosense Webster, Inc. The authors report no other conflicts of interest in this work.

\section{References}

1. Natale A, Reddy VY, Monir G, et al. Paroxysmal AF catheter ablation with a contact force sensing catheter: results of the prospective, multicenter SMART-AF trial. J Am Coll Cardiol. 2014;64(7):647-656.

2. Afzal MR, Chatta J, Samanta A, et al. Use of contact force sensing technology during radiofrequency ablation reduces recurrence of atrial fibrillation: a systematic review and meta-analysis. Heart Rhythm. 2015;12(9):1990-1996.

3. Hussein AA, Barakat AF, Saliba WI, et al. Persistent atrial fibrillation ablation with or without contact force sensing. J Cardiovasc Electrophysiol. 2017;28(5):483-488.

4. Lee G, Hunter RJ, Lovell MJ, et al. Use of a contact force-sensing ablation catheter with advanced catheter location significantly reduces fluoroscopy time and radiation dose in catheter ablation of atrial fibrillation. Europace. 2016;18(2):211-218.

5. Jarman JWE, Panikker S, das M, et al. Relationship between contact force sensing technology and medium-term outcome of atrial fibrillation ablation: a multicenter study of 600 patients. $J$ Cardiovasc Electrophysiol. 2015;26(4):378-384.

6. Sigmund E, Puererfellner H, Derndorfer M, et al. Optimizing radiofrequency ablation of paroxysmal and persistent atrial fibrillation by direct catheter force measurement - a case-matched comparison in 198 patients. Pacing Clin Electrophysiol. 2015;38(2):201-208.
7. Zhou X, Lv W, Zhang W, et al. Impact of contact force technology on reducing the recurrence and major complications of atrial fibrillation ablation: a systematic review and meta-analysis. Anatol $J$ Cardiol. 2017;17(2):82-91.

8. Marijon E, Fazaa S, Narayanan K, et al. Real-time contact force sensing for pulmonary vein isolation in the setting of paroxysmal atrial fibrillation: procedural and 1-year results. J Cardiovasc Electrophysiol. 2014;25(2):130-137.

9. Itoh T, Kimura M, Tomita $\mathrm{H}$, et al. Reduced residual conduction gaps and favourable outcome in contact force-guided circumferential pulmonary vein isolation. Europace. 2016;18(4):531-537.

10. Sciarra L, Golia P, Natalizia A, et al. Which is the best catheter to perform atrial fibrillation ablation? A comparison between standard ThermoCool, SmartTouch, and Surround Flow catheters. J Interv Card Electrophysiol. 2014;39(3):193-200.

11. Gonna H, Domenichini G, Zuberi Z, et al. Initial clinical results with the ThermoCool ${ }^{\circledR}$ SmartTouch ${ }^{\circledR}$ Surround Flow catheter. Europace. 2017;19(8):1317-1321.

12. Naniwadekar A, Joshi K, Greenspan A, Mainigi S. Use of the new contact force sensing ablation catheter dramatically reduces fluoroscopy time during atrial fibrillation ablation procedures. Indian Pacing Electrophysiol J. 2016;16(3):83-87.

13. Miller DL. Make radiation protection a habit. Tech Vasc Interv Radiol. 2018;21(1):37-42.

14. Ko S, Kang S, Ha M, et al. Health effects from occupational radiation exposure among fluoroscopy-guided interventional medical workers: a systematic review. J Vasc Interv Radiol. 2018;29(3):353-366.

15. Picano E, Piccaluga E, Padovani R, Antonio Traino C, Grazia Andreassi M, Guagliumi G. Risks related to fluoroscopy radiation associated with electrophysiology procedures. J Atr Fibrillation. 2014;7(2):1044.

16. Goldsweig AM, Abbott JD, Aronow HD. Physician and patient radiation exposure during endovascular procedures. Curr Treat Options Cardiovasc Med. 2017;19(2):10.

17. Muthalaly RG, John RM, Schaeffer B, et al. Atrial fibrillation ablation safety and complication rates have improved over time. J Cardiovasc Electrophysiol. 2018;29(6):854-860.

18. January CT, Wann LS, Alpert JS. AHA/ACC/HRS guideline for the management of patients with atrial fibrillation: executive summary - a report of the American College of Cardiology/American Heart Association Task Force on Practice Guidelines and the Heart Rhythm Society. J Am Coll Cardiol. 2014;64(21):2246-2280.

19. Rolf S, John S, Gaspar T, et al. Catheter ablation of atrial fibrillation supported by novel nonfluoroscopic 4D navigation technology. Heart Rhythm. 2013;10(9):1293-1300.

20. Ferguson JD, Helms A, Mangrum JM, et al. Catheter ablation of atrial fibrillation without fluoroscopy using intracardiac echocardiography and electroanatomic mapping. Circ Arrhythm Electrophysiol. 2009;2(6):611-619.

21. Reddy VY, Morales G, Ahmed H, et al. Catheter ablation of atrial fibrillation without the use of fluoroscopy. Heart Rhythm. 2010;7(11): 1644-1653.

22. Sommer P, Rolf S, Piorkowski C, et al. Nonfluoroscopic catheter visualization in atrial fibrillation ablation: experience from 375 consecutive procedures. Circ Arrhythm Electrophysiol. 2014;7(5):869-874.

23. Gianni C, Monreal C, Trivedi C, et al. Usefulness of ultrasound-guided femoral vein access in atrial fibrillation ablation. $\mathrm{J} \mathrm{Am} \mathrm{Coll} \mathrm{Cardiol.}$ 2016;67(13):764.

24. Reddy VY, Shah D, Kautzner J, et al. The relationship between contact force and clinical outcome during radiofrequency catheter ablation of atrial fibrillation in the TOCCATA study. Heart Rhythm. 2012;9(11): 1789-1795.

25. Kuck KH, Reddy VY, Schmidt B, et al. A novel radiofrequency ablation catheter using contact force sensing: Toccata study. Heart Rhythm. 2012;9(1):18-23. 
Pragmatic and Observational Research

\section{Publish your work in this journal}

Pragmatic and Observational Research is an international, peer-reviewed, open access journal that publishes data from studies designed to reflect more closely medical interventions in real-world clinical practice compared with classical randomized controlled trials (RCTs). The manuscript management system is completely online and includes a very quick and fair peer-review

Submit your manuscript here: https://www.dovepress.com/pragmatic-and-observational-research-journal

\section{Dovepress}

system. Visit http://www.dovepress.com/testimonials.php to read real quotes from published authors. 